\title{
Effect of Alkaloids Belong to $\beta$-Carbolines Family in Peganum Harmala on Cancer Cells
}

\section{ART ICLE INF O}

\section{Article Type}

Systematic Review

\section{Authors}

Roostaee Z* $P h D$

\section{How to cite this article} Roostaee Z. Effect of Alkaloids Belong to $\beta$-Carbolines Family in Peganum Harmala on Cancer Cells. Sarem Journal of Reproductive Medicine. 2018;2(2):73-78
*Sarem Fertility \& Infertility Research Center (SAFIR), Sarem Women's Hospital, Tehran, Iran

\section{Correspondence}

Address: Sarem Women's Hospital, Basij Square, Phase 3, Ekbatan Town, Tehran, Iran. Postal Code: 1396956111

Phone: +98 (21) 44670888

Fax: +98 (21) 44670432

zrmj1394@gmail.com

\section{Article History}

Received: January 25, 2017

Accepted: April 20, 2017

ePublished: June 15, 2018

\section{A B S T R A C T}

Introduction As a herb, Peganum harmala has been important in traditional medicine for a long time. Some studies have shown the inhibitory effect of many plant compounds, including alkaloids belonging to $\beta$-carboline family, on cancer cells. This plant contains important alkaloids, such as Harmaline, Harmine, Harmane, and some other components of -carboline family. The literature in this field shows that various concentrations of the alkaloids are effective in treating various diseases, including inhibition of cancer cells. The aim of this study was to introduce Peganum harmala as one of the natural potentials of Iran in relation to herbs and its pharmacological effects. In this study, the keywords Peganum harmala, pathway of betacarbolins in the human body, betacarbolins, Toxicity, and Anti-cancer were searched in Elsevier, PubMed, and Google Scholar databases and the related articles were provided. Then, in advanced search, the pharmacological effects of Peganum harmala, apoptosis, and the mechanism of analgesic effects were searched both in Persian and in English. Finally, 30 articles related to Peganum harmala in traditional medicine were downloaded, translated, and all articles underwent content analysis.

Conclusion As one of the most important herbs with valuable alkaloids belonging to the $\beta$-carboline family, African rue "Peganum harmala" can produce compounds against cancer cells that can be effective in the treatment of cancer. The review of the studies has also shown that the plant extract is effective in preventing the progression of cancer cells.

Keywords Peganum Harmala; $\beta$-Carboline; Alkaloids; Pharmacology; Cancer

\section{I T A T I O N L IN K S}

[1] Interaction between cytotoxic effects of Xanthomicrol and Noscapine on PC12 cells [2] Anti tumoral and differentiayion effects of alkaloids of Harmine and Harmaline on leukaemic cells treated with atra and G-CSF [3] Beta-Carboline alkaloids in peganum harmala and inhibition of human monoamine oxidase (MAO) [4] Stereospecific biotransformation of $( \pm$ ) phenylethyl propionate by cell cultures of peganum harmala L [5] Distribution and medicinal importance of Peganum harmala [6] Toxicity of peganum harmala: Review and a case report [7] Acute toxicological studies on the extract of Iraqi Peganum harmala in rats [8] Synthesis of beta-carboline derivatives [9] Antibacterial activity of different parts of Peganum harmala L. growing in Iran against multi-drug resistant bacteria [10] Effect of Harmaline on seizure induced byamygdala kindling in rats [11] Preliminary studies on the hypoglycemic effect of Peganum harmala L. seeds ethanol extract on normal and streptozotocin induced diabetic rats [12] Antitumor agents 201. 1 Cytotoxicity of harmine and $\beta$-carboline analogs [13] Antitumor and neurotoxic effects of novel harmine derivatives and structure-activity relationship analysis [14] Antitumor properties and toxicity effects of Peganum harmala L.(Zygophyllaceae) [15] Beta-Carboline alkaloids and essential tremor: Exploring the environmental determinants of one of the most prevalent neurological diseases [16] An overview of cognitive aspects of $\beta$-carbolines [17] Effects of harmane, norharmane and harmine on apomorphine-induced pecking behavior in chick [18] Hemolytic effect of total alkaloids from the seeds of Peganum harmala in vitro on erythrocytes of ruminants: Sheep, cattle and goats [19] Apoptosis: Programmed cell death [20] Molecular and Cellular basis of human cancer [21] Harmine activates intrinsic and extrinsic pathways of apoptosis in B16F10 melanoma [22] A review on medicinal importance, pharmacological activity and bioanalytical aspects of beta-carboline alkaloid "Harmine" [23] Cytotoxic indole alkaloids against human leukemia cell lines from the toxic plant Peganum harmala [24] Acute promyelocytic leukemia: Evolving therapeutic strategies [25] Vasorelaxant effects of harmine and harmaline extracted from Peganum harmala L. seed's in isolated rat aorta [26] Effects of aqueous and ethanolic extracts of Peganum harmala L seeds on lipids profile in rats [27] Effects of opiate receptor agonists and antagonists on spontaneuse seizure activity in hipocampal slices [28] Plants as a source of anti-cancer agents 
كشورهاى مختلف بنا بر آداب و رسوم آنان متفاوت است؛ بهاطور

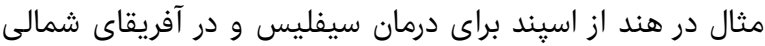

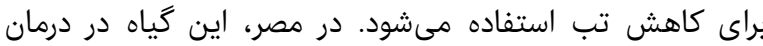

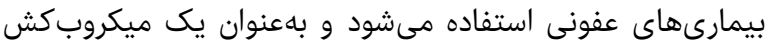

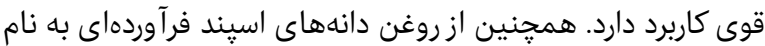

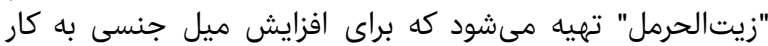

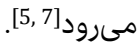
در جنگ جهانى دوم از آلكالوئيد هارمين موجود در اسيند بهاعنوان

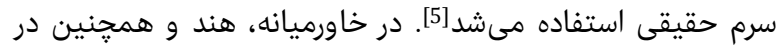

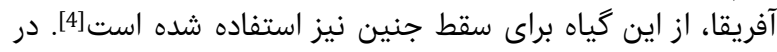

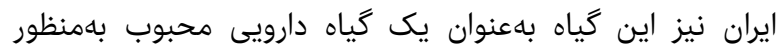

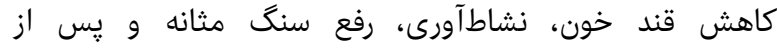

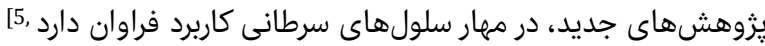

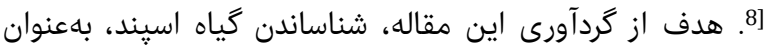

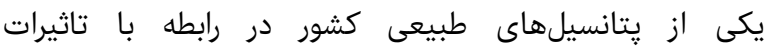

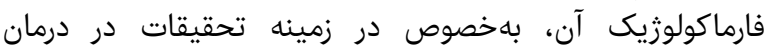

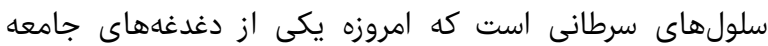

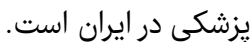

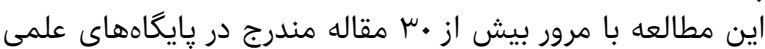

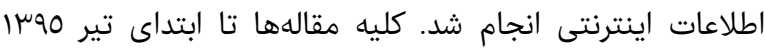

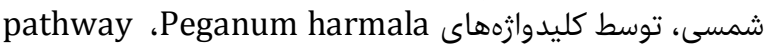
،betacarbolins ،of betacarbolins in human body ، Elsivier Toxicity and Anti-cancer

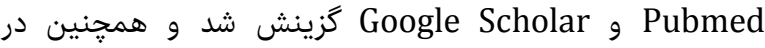

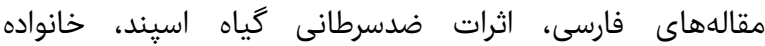

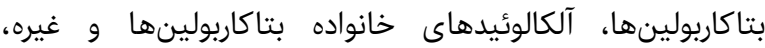

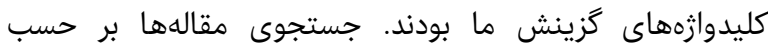

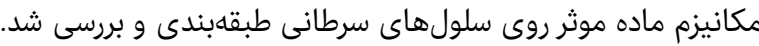

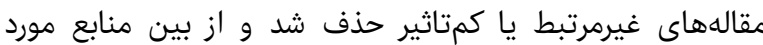

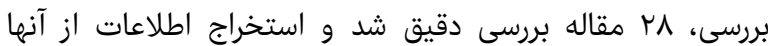

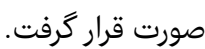

Harmaline

اثر آلكالوئيدهاى متعلق به خانواده بتاكاربولينها

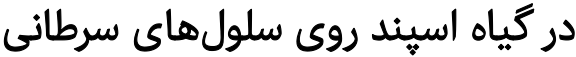

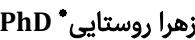

مركز تحقيقات بارورى و نابارورى صارم، بيمارستان فوق تخصصى صارم، تهران،

جكيده

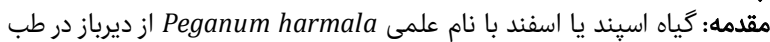

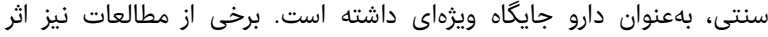

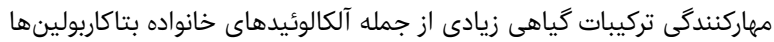

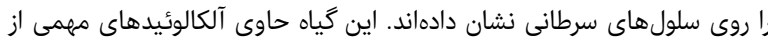

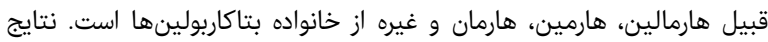

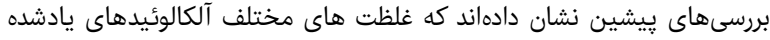

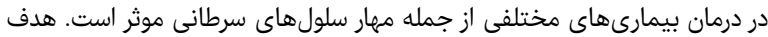

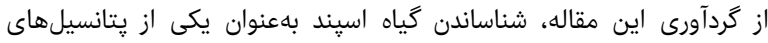

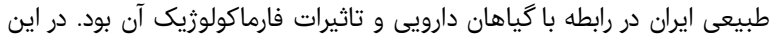
pathway of مطالعه ابتدا كليدوازههاى Peganum harmala، Toxicity and Anti- ،betacarbolins ،betacarbolins in human body cancer

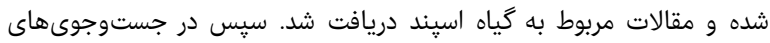

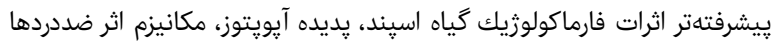

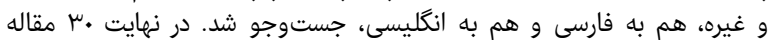

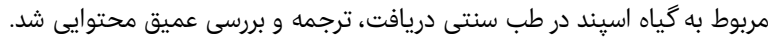

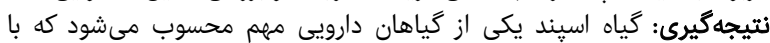

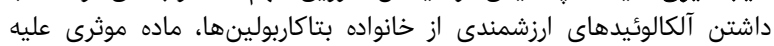

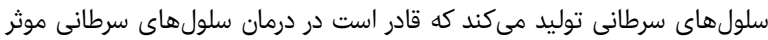

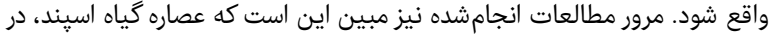

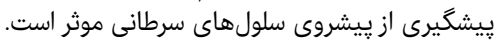
كليدوارهها: كَياه اسيند، آلكالوئيد، بتاكاربولينها، فراني مارماكولوزى، سرطان

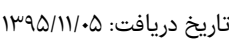

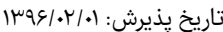
"نويسنده مسئول: zrmj1394@gmail.com

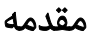

گياه اسفند يا اسيند، با نام علمى Peganum harmala و نام

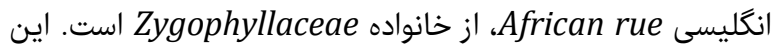

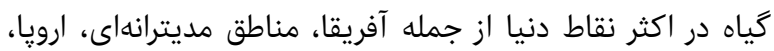

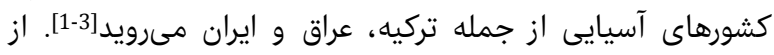

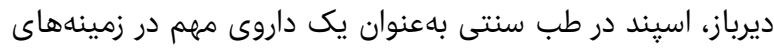

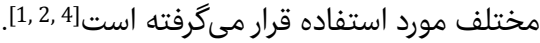

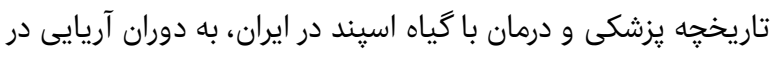

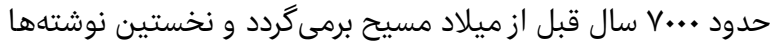

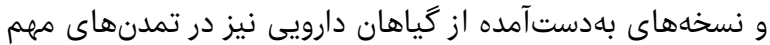

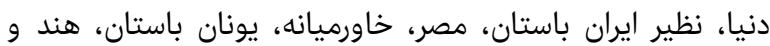

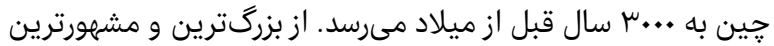

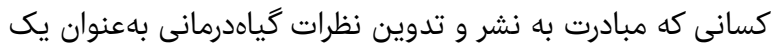

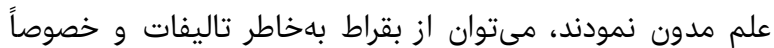

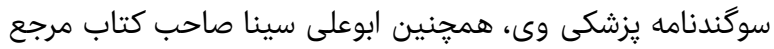

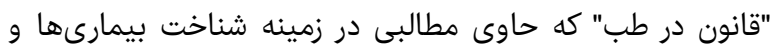

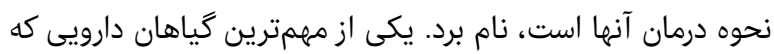

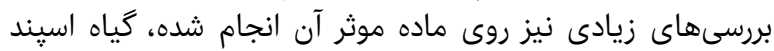

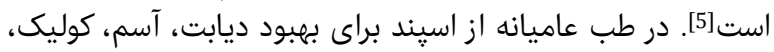

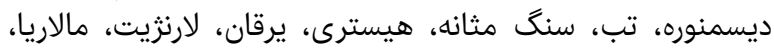

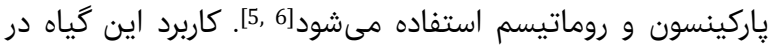




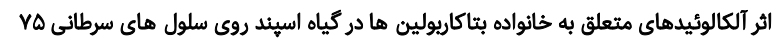

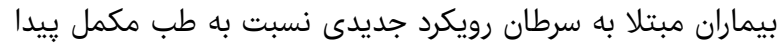

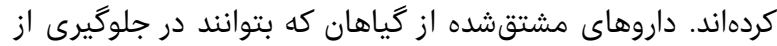

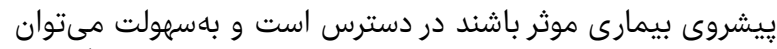

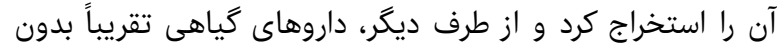

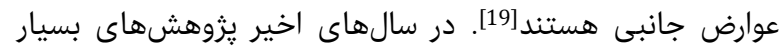

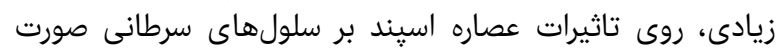

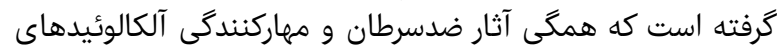

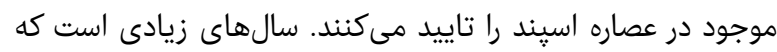

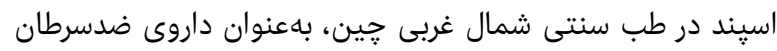

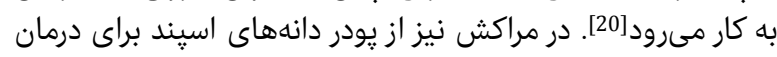

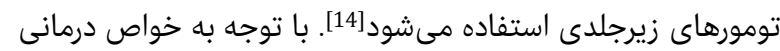

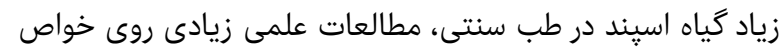

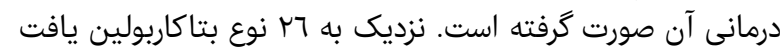

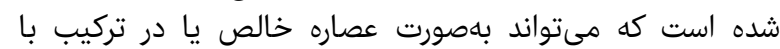

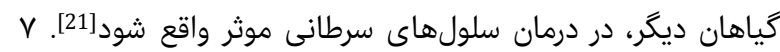

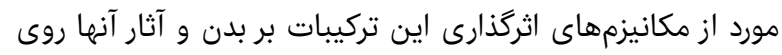

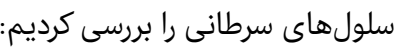

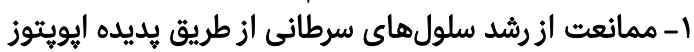

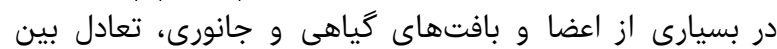

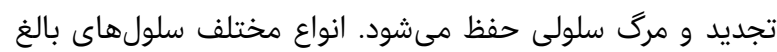

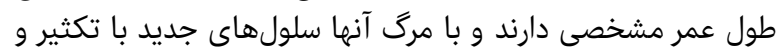

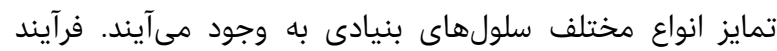

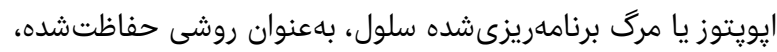

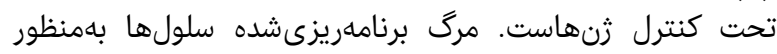

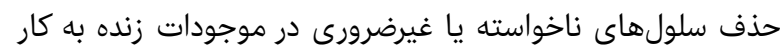

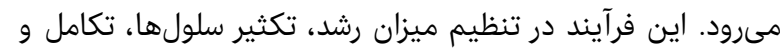

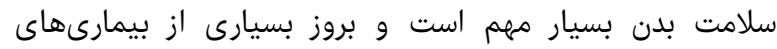

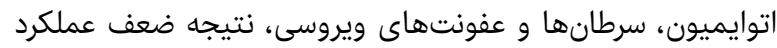

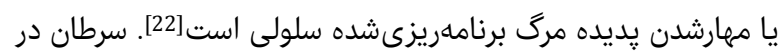

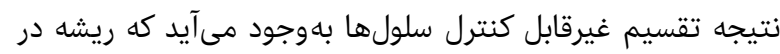

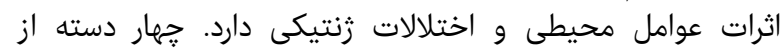

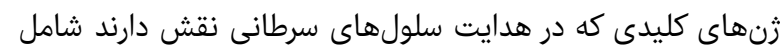

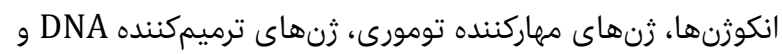

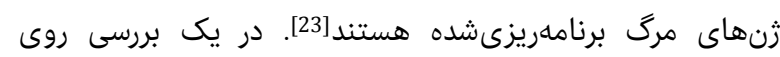

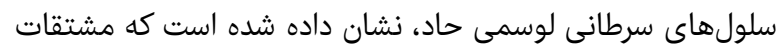

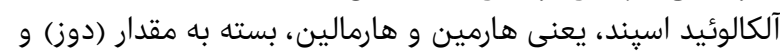

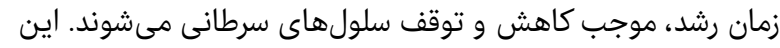

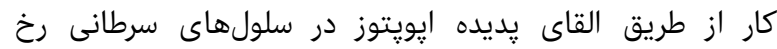

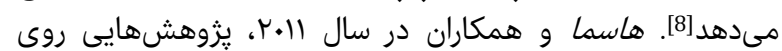

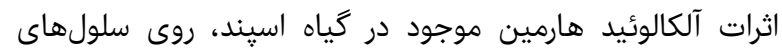

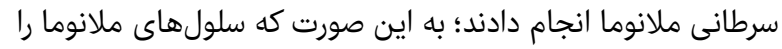

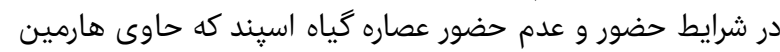

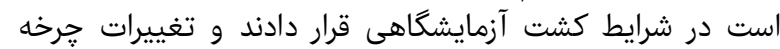

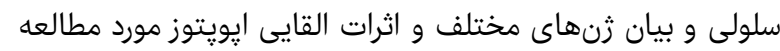

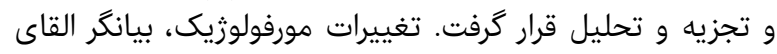

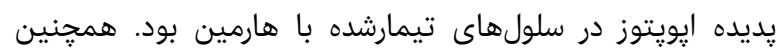

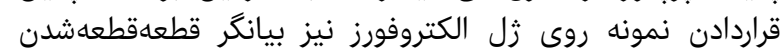

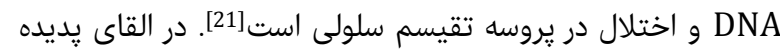

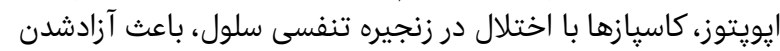

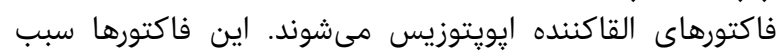

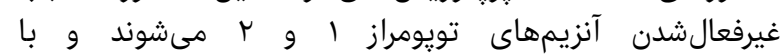

گَياه اسيند حاوى آلكالوئيدهاى مهمى است كه بهترتيب اولويت

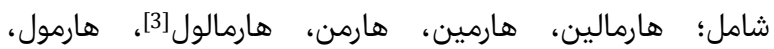

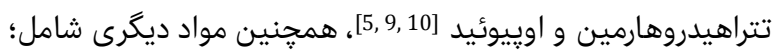

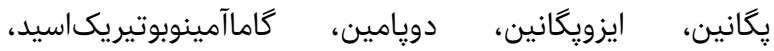

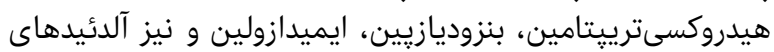

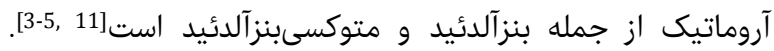

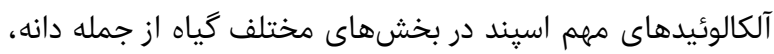

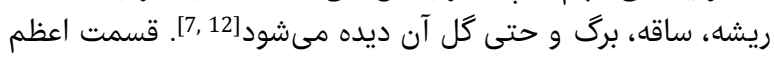

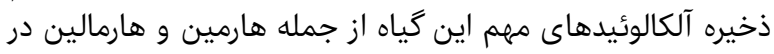

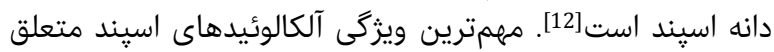

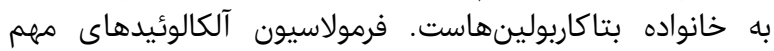

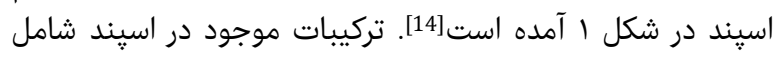

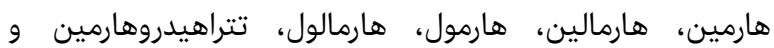

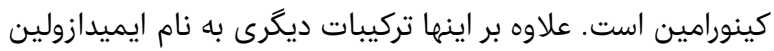

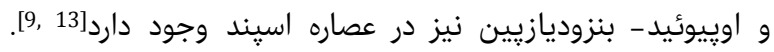

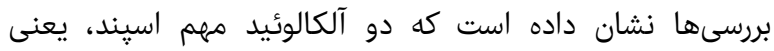

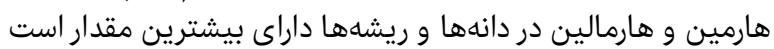

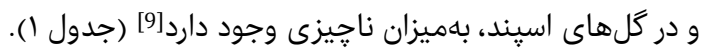

\begin{tabular}{|c|c|c|c|c|c|}
\hline ريشه & ساقه & برى & إنل & 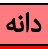 & آلكالوئيدهاى اسيند \\
\hline+ & + & - & - & + & Harman alkaloids (Harmine) \\
\hline+ & + & - & - & + & $\begin{array}{c}\text { Harmaline } \\
\text { Dehydroharmine }\end{array}$ \\
\hline - & + & - & + & + & $\begin{array}{l}\text { Quinzoline derivative, de } \\
\text { vasicine (Peganine) }\end{array}$ \\
\hline- & - & - & - & - & $\begin{array}{l}2,3 \text { trimethylene } \& 4 \\
\text { quinazolone }\end{array}$ \\
\hline - & + & - & - & + & $\begin{array}{l}\text { 1,2,3- hydroxytrimethylene } \\
\text { quinqzolone (Harmalol) }\end{array}$ \\
\hline - & - & - & - & + & Harmalidine $\beta$ carboline \\
\hline - & - & - & - & + & Harmaline \\
\hline+ & + & + & - & + & Pegamine \\
\hline+ & + & + & - & + & Vascinones \\
\hline
\end{tabular}

\section{خانواده بتاكاربولينها}

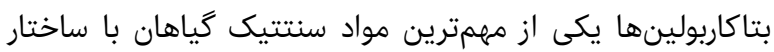

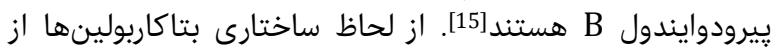

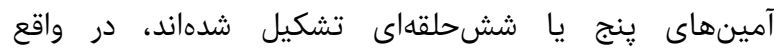

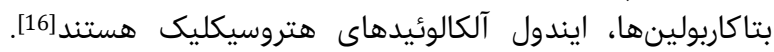

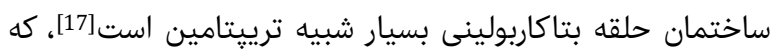

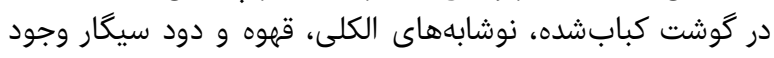

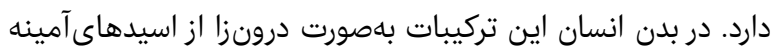

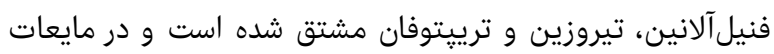

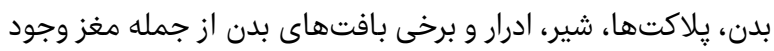

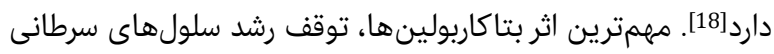

است.

\section{اثر آلكالوئيدهاى خانواده بتاكاربولينهاى اسيند روى سلولهاى سرطانى ائى}

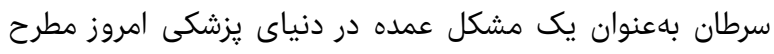

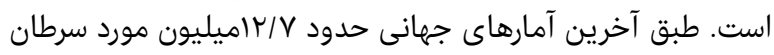

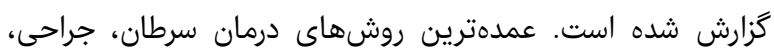

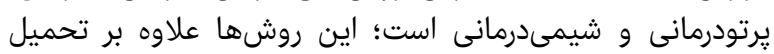

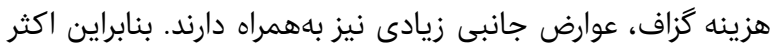




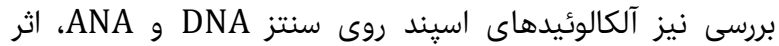

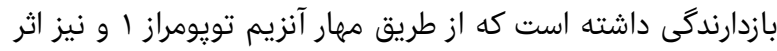

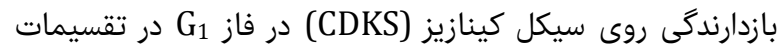

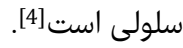

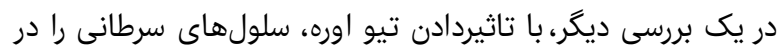

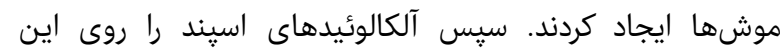

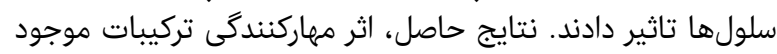

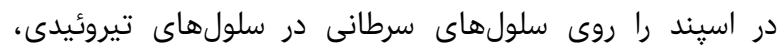

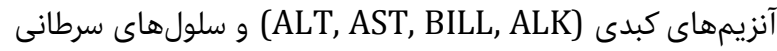

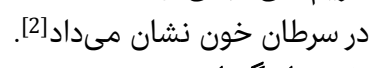

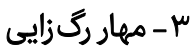

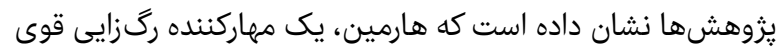

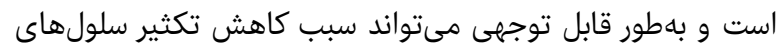

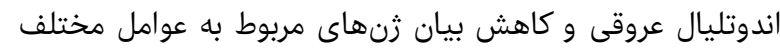

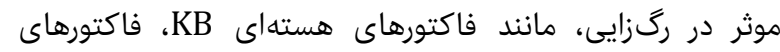

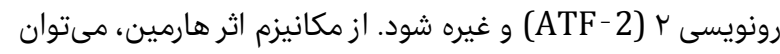

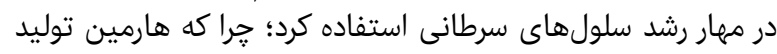

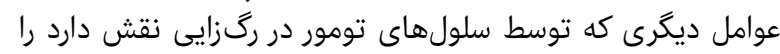
كاهش مىدهد و از اين طريق سبب مهار ركار رگزايى در تومور

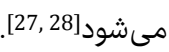
ع ع اثرات آنتىاكسيدانى اسيند

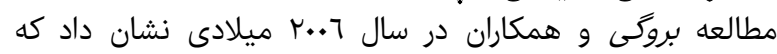

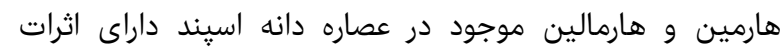

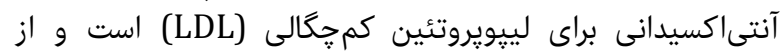

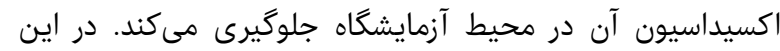

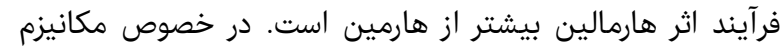

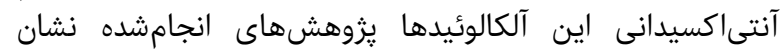

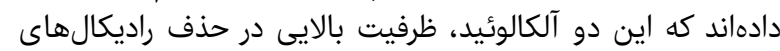

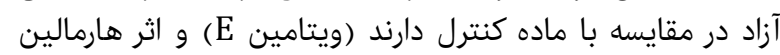

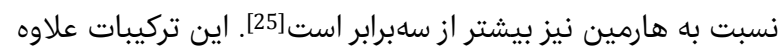

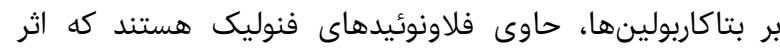

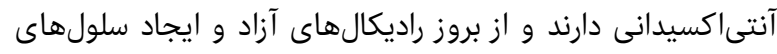

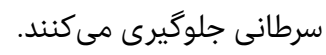

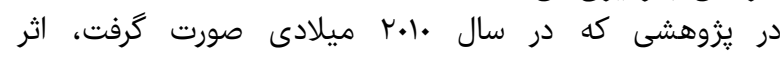

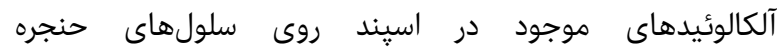

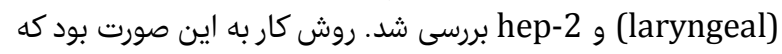

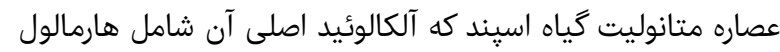

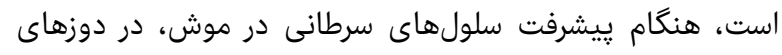

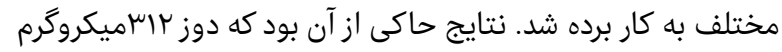

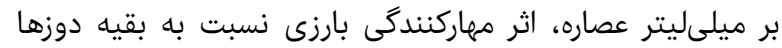

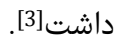

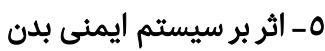

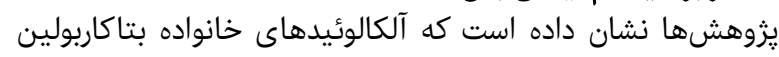

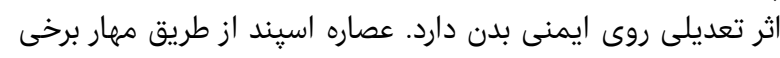

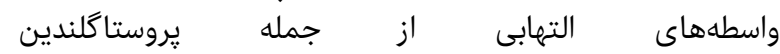

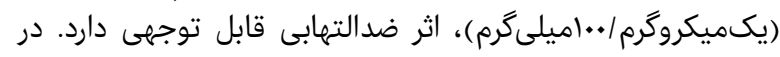

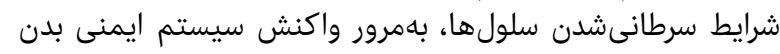

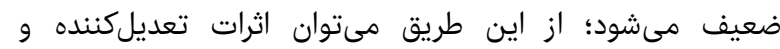

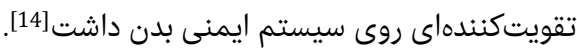

7- ت ضددرد

يزوهشهايى روى برخى تركيبات اسيند مانند هارمن و هارمين و
قطعه قطعهكردن DNA، در همانندسازى آن اختلال ايجاد مىكنيند.

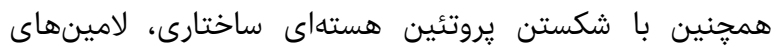
غشاى هسته توسط كاسياز 7 تخريب مئشئ هوند

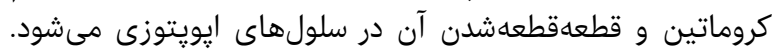

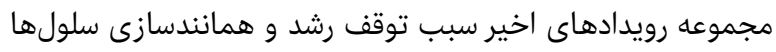

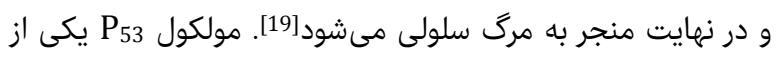

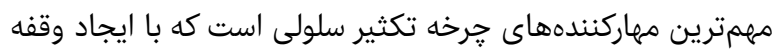

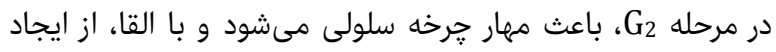

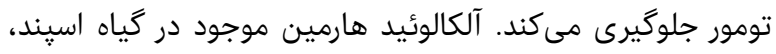

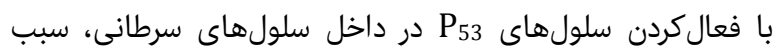

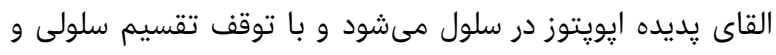

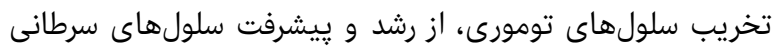

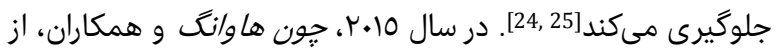

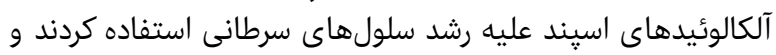

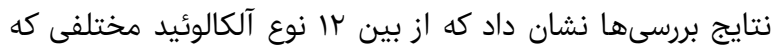

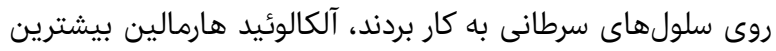

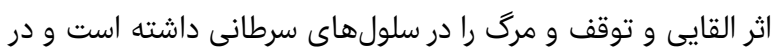

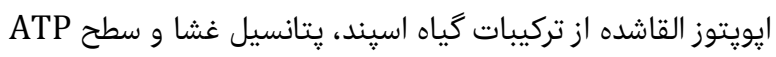
ميتوكندرى، بهطور جشمكيرى در سلولهاى سرطان لينان خون كاهش و

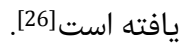

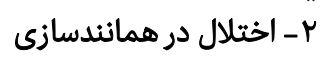

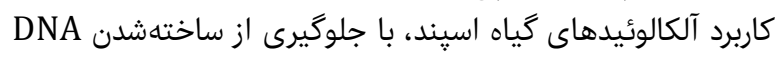

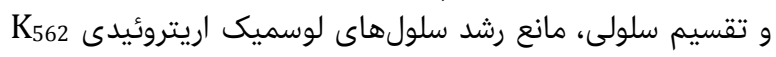

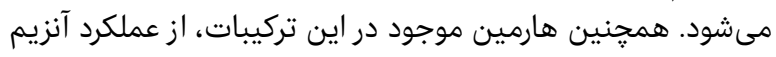

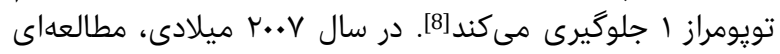

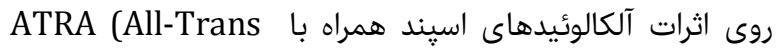
و و ويتامين Detinoic Asid)

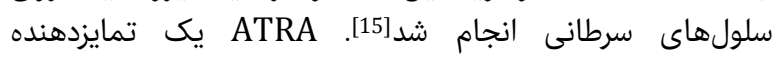

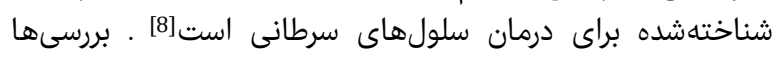

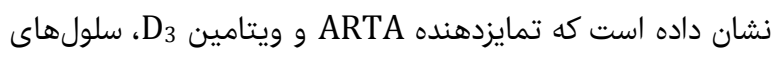

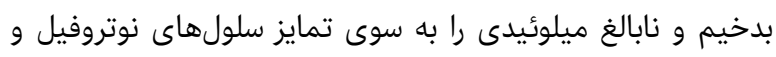

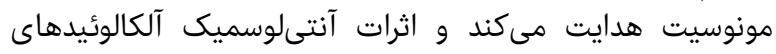

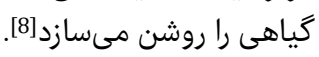

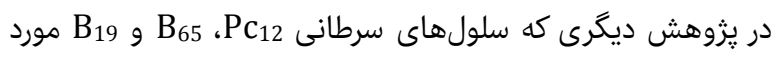

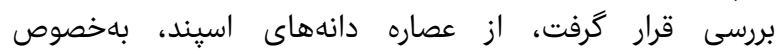

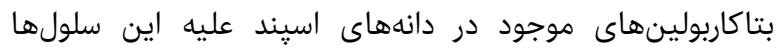

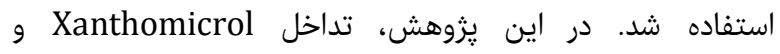
Soscapine

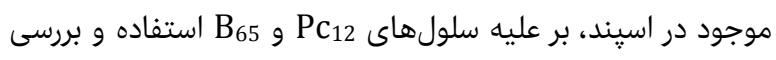

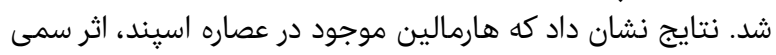

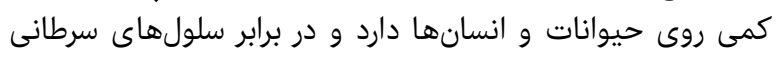

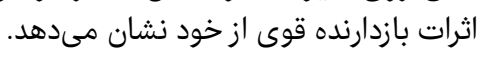

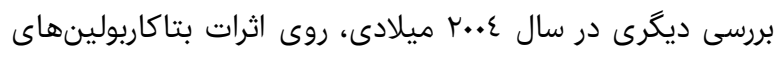

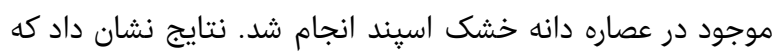

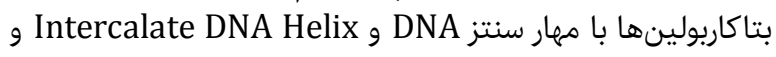

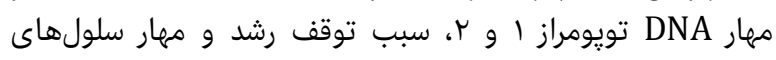

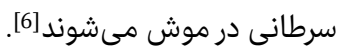

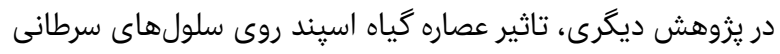
Vcp-med sarcoma Med-mek carcinoma ،Vcp-med

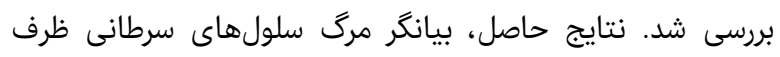

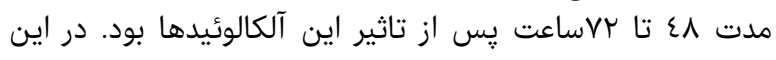


نتيجه

كياه اسيند يكى از مهمترين كَياهان دارويى است كه در سطح ايران

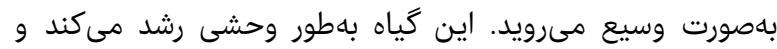

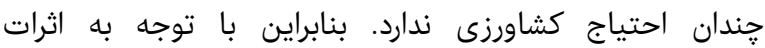

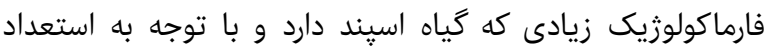

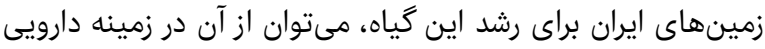

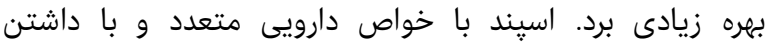

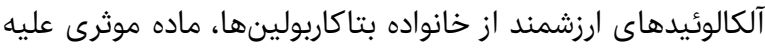

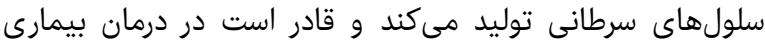

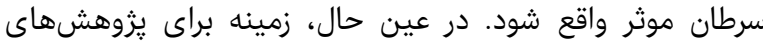
بيشتر در خصوص تاثيرات اين كياه فراهم است إن.

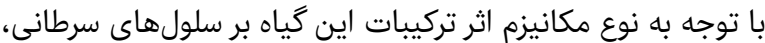

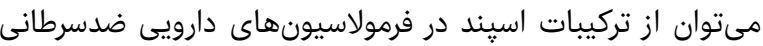

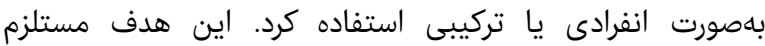

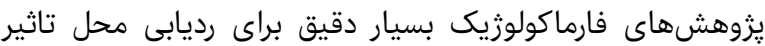
تركيبات اين كياه است.

تشكر و قدرداني: موردى از سوى نويسنده كزارش نشده است.

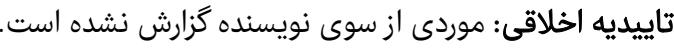
تعارض منافع: موردى از سوى نويسنده كزارش نشارئ نشاره است. منابع مالى: موردى از سوى نويسنده كزارش نشار نشده است. سهم نويسندان: زهرا روسنايى (نويسنده اول)، تمام امور مربوط أنوان به مقاله توسط ايشان انجام شده است (••ام).

منابع

1- Ebrahimi SA, Kenari FJ. Interaction between cytotoxic effects of Xanthomicrol and Noscapine on PC12 cells. J Genes Microb Immun. 2013;1-8. Available From: http://www.ispacs.com/journals/jgmi/2013/jgmi00005/.

2- Zaker F. Anti tumoral and differentiayion effects of alkaloids of Harmine and Harmaline on leukaemic cells treated with atra and G-CSF. Razi J Med Sci. 2004;10(38):869-75.

3- Herraiz T, Gonzalez D, Ancín-Azpilicueta C, Arán VJ, Guillén H. Beta-Carboline alkaloids in peganum harmala and inhibition of human monoamine oxidase (MAO). Food Chem Toxicol. 2010;48(3):839-45.

4- Asghari G, George Br. Stereospecific biotransformation of $( \pm)$ phenylethyl propionate by cell cultures of peganum harmala L. Iran Biomed J. 2002;6(1):43-46.

5- Aslam N, Wani AA, Nawchoo IA, Bhat MA. Distribution and medicinal importance of Peganum harmala. Int J Adv Res. 20142(2)751-5.

6- Mahmoudian M, Jalipour H, Dardashti PS. Toxicity of peganum harmala: Review and a case report. Iran J Pharmacol Ther. 2002;1(1):1-4.

7- Muhi-eldeen Z, Al-Shamma KJ, Al-Hussainy TM, AlKaissi EN, Al-Daraji AM, Ibrahim H. Acute toxicological studies on the extract of Iraqi Peganum harmala in rats. Europ J Sci Res. 2008;22(4):494-500.

8- Bernardo C, Olival ACD, Ribeiro A, Rodrigues L, Esteves AP, Campos AM. Synthesis of beta-carboline derivatives [Internet]. Santiago: Universidade de Santiago de Compostela; 2017. [Update 2017 May 25, Cited 2017]. Avilable From: www.usc.es/congresos/ecsoc/16/hall_a_GOS/a027/inde x.pdf.

9- Darabpour E, Motamedi H, Poshtkouhian Bavi A, Nejad
اثر مهاركنندگى اين تركيبات بر سندروم محروميت القاشده

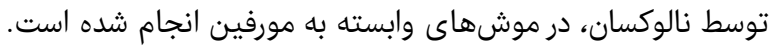

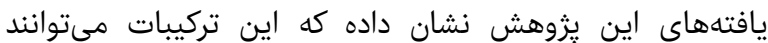

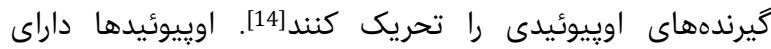

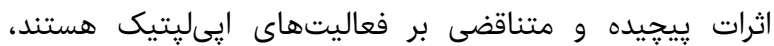

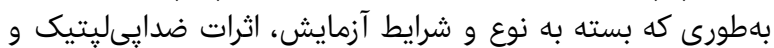

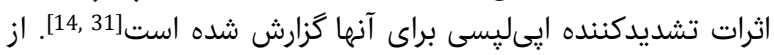

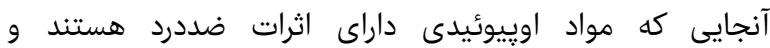

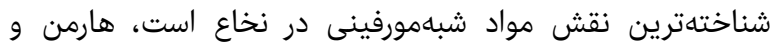

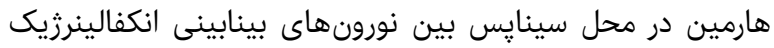

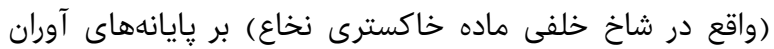

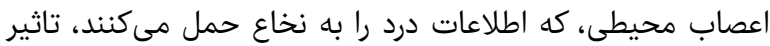

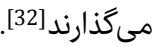

- V اثر روى آنزيم مونوآميناكسيداز (MA0)

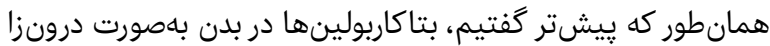

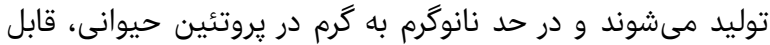

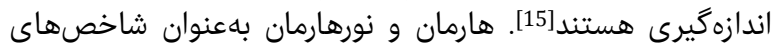

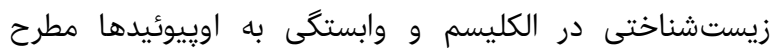

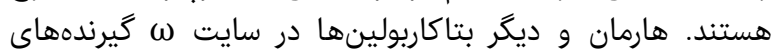

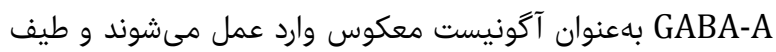

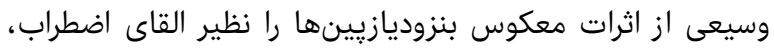

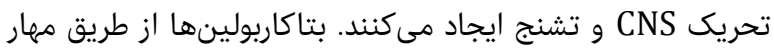

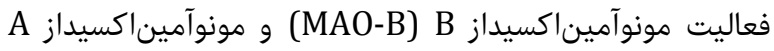

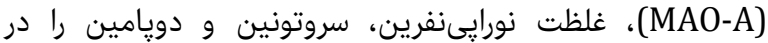

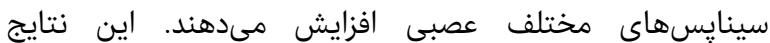

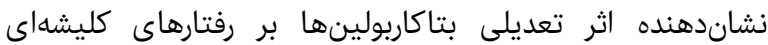

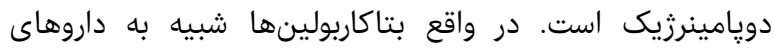

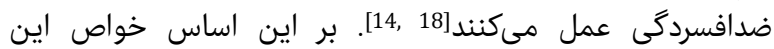

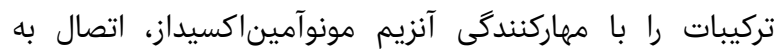

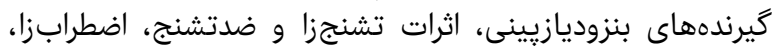

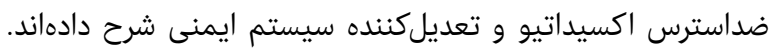

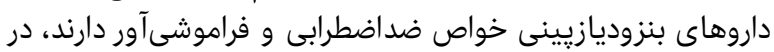

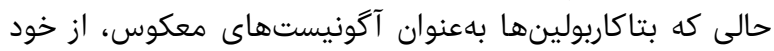

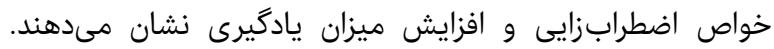

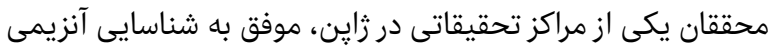

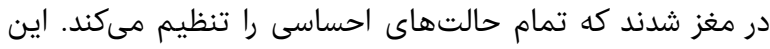

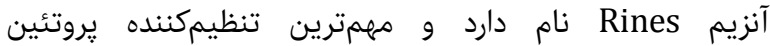

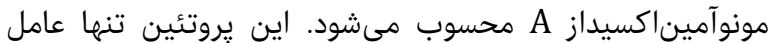
تنظيمكننده احساسات و خلق و خوى افرين افراد است. اين ليروتئين

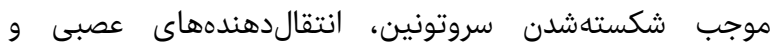

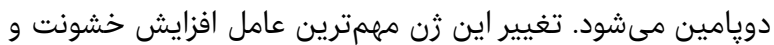

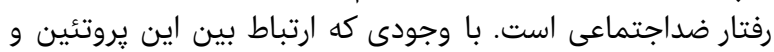

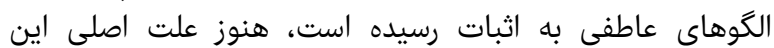

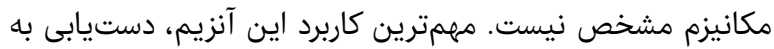

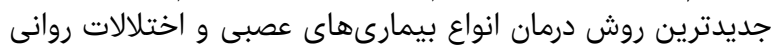

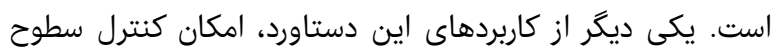

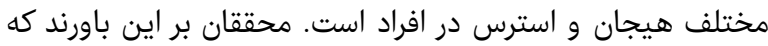

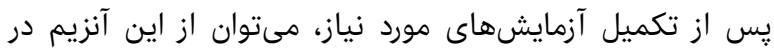

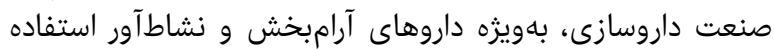
كرد. نتايج كامل اين تحقيقات در شماره اخير نشرئ داروايه ارملئ علوم اعصاب (Neuroscience) 
2013;3(6):53-9.

19- Honardoost M, Solimanjahi H, Farzad R. Apoptosis: Programmed cell death. J Qazvin Univ Med Sci. 2013;17(3):48-57.

20- Parsa N. Molecular and Cellular basis of human cancer. J Cell Tissue. 2012;2(4):365-76.

21- Hamsa TP, Kuttan G. Harmine activates intrinsic and extrinsic pathways of apoptosis in B16F-10 melanoma. Chin Med. 2011;6:11.

22- Patel K, Gadewar M, Tripathi R, Prasad S, Patel DK. A review on medicinal importance, pharmacological activity and bioanalytical aspects of beta-carboline alkaloid "Harmine". Asian Pac J Trop Biomed. 2012;2(8):660-4.

23- Wang C, Zhang Z, Wang Y, He X. Cytotoxic indole alkaloids against human leukemia cell lines from the toxic plant Peganum harmala. Toxins (Basel). 2015;7(11):4507-18.

24- Tallman MS, Nabhan C, Feusner JH, Rowe JM. Acute promyelocytic leukemia: Evolving therapeutic strategies. Blood. 2002;99(3):759-67.

25- Berrougui H, Martín-Cordero C, Khalil A, Hmamouchi M, Ettaib A, Marhuenda E, et al. Vasorelaxant effects of harmine and harmaline extracted from Peganum harmala L. seed's in isolated rat aorta. Pharmacol Res. 2006;54(2):150-7.

26- Hasanzadeh Tahery M, Hasanpour fard M, Rabiee N, Ghoreishe AR, Ravanbakhsh N. Effects of aqueous and ethanolic extracts of Peganum harmala L seeds on lipids profile in rats. J Birjand Univ Med Sci. 2013;20(2):10814.

27- Esmaeili MH, Haghdost H, Gheybea N. Effects of opiate receptor agonists and antagonists on spontaneuse seizure activity in hipocampal slices. koomesh. 2007;9(1):27-32.

28- Cragg GM, Newman DJ. Plants as a source of anticancer agents. J Ethnopharmacol. 2005;100(1-2):72-9.
S, Mansour S. Antibacterial activity of different parts of Peganum harmala L. growing in Iran against multi-drug resistant bacteria. EXCLI J. 2011;10:252-263.

10- Alenajaf AAF, M\%A Moghimi, A\%A Mohebi, E\%A Mohammad-Zadeh, M. Effect of Harmaline on seizure induced byamygdala kindling in rats. J North Khorasan Univ Med Sci. 2015;6(4):697-706.

11- Singh AB, Chaturvedi J, Narender T, Srivastava AK. Preliminary studies on the hypoglycemic effect of Peganum harmala L. seeds ethanol extract on normal and streptozotocin induced diabetic rats. Indian J Clin Biochem. 2008;23(4):391-3.

12- Ishida J, Wang $\mathrm{H}-\mathrm{K}$, Bastow $\mathrm{KF}, \mathrm{Hu} \mathrm{C}-\mathrm{Q}$, Lee $\mathrm{K}-\mathrm{H}$. Antitumor agents 201. 1 Cytotoxicity of harmine and $\beta$ carboline analogs. Bioorg Med Chem Lett. 1999;9(23):3319-24.

13- Chen Q, Chao R, Chen H, Hou X, Yan H, Zhou S, et al. Antitumor and neurotoxic effects of novel harmine derivatives and structure-activity relationship analysis. Int J Cancer. 2005;114(5):675-82.

14- Lamchouri F. Antitumor properties and toxicity effects of Peganum harmala L.(Zygophyllaceae). Plant Sci Today. 2014;1(4):192-5.

15- Louis ED, Zheng W. Beta-Carboline alkaloids and essential tremor: Exploring the environmental determinants of one of the most prevalent neurological diseases. Sci World J. 2010;10:1783-94.

16- Heshmati P, Nasehi M, Zarrindast MR. An overview of cognitive aspects of $\beta$-carbolines. J Paramed Sci. 2013;5(1):99-104.

17- Farzin DAS, E. Effects of harmane, norharmane and harmine on apomorphine-induced pecking behavior in chick. J Mazandaran Univ Med Sci. 2009;19(70):1-8.

18- Mahdeb N, Mayouf S, Boukhari F, Souilah S, Bouzidi A. Hemolytic effect of total alkaloids from the seeds of Peganum harmala in vitro on erythrocytes of ruminants: Sheep, cattle and goats. Asian J Plant Sci Res. 